\title{
Colorimetric and refractometric measurements of carbon dioxide
}

\author{
C. Gouveia ${ }^{1,2}$, A. Markovics ${ }^{3}$, J. M. Baptista ${ }^{1,2}$, B. Kovács ${ }^{3,4}$, P. A. S. Jorge ${ }^{1}$ \\ ${ }^{1}$ INESC Porto, Rua do Campo Alegre 687, 4169-007 Porto, Portugal \\ ${ }^{2}$ Centro de Competências de Ciências Exactas e de Engenharia, Universidade da Madeira, \\ Campus da Penteada, 9000-390 Funchal, Portugal \\ ${ }^{3}$ South Trans Danubian Research Cooperative (DDKKK) \\ ${ }^{4}$ Department of General and Physical Chemistry, Faculty of Sciences, University of Pécs, \\ Ifjúság Útja 6, H-7624, Pécs, Hungary
}

\begin{abstract}
In this work, a polymeric sensitive layer based on the acid-base equilibrium of phenol and of its derivative p-nitro-phenol is presented for carbon dioxide measurements. Thin films casted on glass slides were tested, using a LED source $(\lambda \mathrm{c}$ at $410 \mathrm{~nm}$ ) and an Ocean Optics USB4000 spectrometer, in the $0 \%$ to $15.25 \% \mathrm{CO}_{2}$ concentrations range, showing a $40 \%$ maximum transmittance variation with a 51s response time and a $0.15 \%$ resolution. Preliminary results indicate that $\mathrm{CO}_{2}$ also induces refractive index changes in the sensitive layer. Using a fiber based interferometric setup, a $\mathrm{CO}_{2}$ dependent refractive index change of $\sim 0.045 \mathrm{RIU}$ was observed, in the $0 \%-90 \% \mathrm{CO}_{2}$ concentration range.
\end{abstract}

Keywords: $\mathrm{CO}_{2}$ sensor, Absorption, Interferometry, Refractometry, Optical Fibers

\section{INTRODUCTION}

The measurement of chemical parameters using optical sensors is an expanding area of research with growing importance, especially in environmental and industrial applications[1]. The development of new sensitive membranes, which changes their optical properties in presence of an analyte, in combination with optical fibre technologies allows to create powerful tools for in situ and real time monitoring of chemical and biological species [2, 3]. Real time determination of $\mathrm{CO}_{2}$ concentration is an important issue in diverse fields, such as chemical and clinical analysis $[4,5]$, food industry (eg. aquaculture) and environmental monitoring [6,7]. Specifically in aquaculture systems, the level of $\mathrm{CO}_{2}$ is an important issue for fish health and development. Elevated dissolved $\mathrm{CO}_{2}$ concentration in the water will induce stress responses in fish causing significant physiological disturbances that can result in reduced growth and poor feed conversion[8].

Several fibre optic based solutions have been proposed for carbon dioxide detection mostly relaying in either colorimetric or fluorescence based mechanisms. Segawa et al [9] presented a $\mathrm{CO}_{2}$ fiber sensor based on the $\mathrm{pH}$ indicator dye thymol blue, immobilized in a sol-gel matrix. The optical attenuation varied according with the $\mathrm{CO}_{2}$ concentration ue to absorption change of a indicator dye induced by the carbon dioxide induced acidification. Amao and Nakamura [10], on the other hand, demonstrated a $\mathrm{CO}_{2}$ sensor based in luminescence intensity changes of tetraphenylporphyrin (TPP) due to absorption changes of a co-immobilized $\mathrm{pH}$ indicator dye. Several other solutions based in $\mathrm{pH}$ indicators have been proposed using luminescent based schemes [7]. Nevertheless configurations using indicator dyes are prone to suffer from leaching and photobleaching effects. In addition, working in the visible range imposes some limitations in applications where remote and multipoint detection are a priority. Using direct absorption spectroscopy of the gas absorption lines in the mid infrared is also a reliable sensing mechanism. Nevertheless sensing at these wavelengths $(\sim 4,6 \mu \mathrm{m})$ requires the use of special chalcogenide glass optical fibers[11]. Using higher harmonics of these vibrational absorption lines is possible nevertheless for instance at $1.57 \mu \mathrm{m}$. Orghici et al [12], showed that carbon dioxide concentration could be measured using an evanescent field sensor based in a quartz glass multimode fibre in this wavelength range. However, this approach required DFB laser sources, relatively long interaction lengths and was very sensitive to contamination of the fiber surface.

In this work, a polymer based sensitive layer for carbon dioxide measurement is presented. The sensing layer is based on the acid-base equilibrium of phenol and of its derivative p-nitro-phenol, which, in the presence of $\mathrm{CO}_{2}$, are prone to protonation introducing absorption and refractive index changes. The new sensitive layers were casted on glass slides and characterized by absorption spectroscopy in the visible range $(\lambda c$ at $410 \mathrm{~nm})$ and its refractive index was also

International Conference on Applications of Optics and Photonics, edited by Manuel F. M. Costa,

Proc. of SPIE Vol. 8001, 80013C · C 2011 SPIE · CCC code: 0277-786X/11/\$18 · doi: 10.1117/12.892091

Proc. of SPIE Vol. $800180013 \mathrm{C}-1$ 
monitored using an interferometric scheme at $1550 \mathrm{~nm}$. The dual effect observed indicates the possibility of $\mathrm{CO}_{2}$ determination using either mechanism. Therefore it can be possible to use this sensitive layer with fiber optics in visible range using standard colorimetric measurement setup or in highly sensitive refractometric configurations based in standard telecom optoelectronic components. The latter approach is quite promising in terms of multiplexing and remote monitoring capabilities.

\section{EXPERIMENTAL RESULTS}

\subsection{Sensing layer chemistry}

The sensing chemistry is based on the acid-basic equilibrium of phenol and of its derivative p-nitro-phenol. They are kept in their deprotonated form in the sensing membrane. In the presence of carbon dioxide, hydrogen carbonate is formed that partially protonates the phenols. The hydroxyl group is involved in protolitic reactions that modify the charge distribution in the molecule. As result of the interaction with carbon dioxide, a change in absorption and in their refractive index is expected due to the delocalized electrons in the aromatic ring of these compounds. A quaternary ammonium compound, didodecyl-dimethyl-ammonium hydroxide (DDMA) was used to deprotonate the phenols, preparing them for being protonated by the analyte.

The sensing layers were prepared of a $5 \%(\mathrm{~m} / \mathrm{m})$ polyurethane hydrogel (D4) dissolved in ethanol solution. Then $10 \mu 1$ of $0.1 \mathrm{M}$ ethanolic p-nitrophenol and $20 \mu \mathrm{l}$ of $0.1 \mathrm{M}$ ethanolic DDMA solution were added to $1 \mathrm{ml}$ of polymer solution. In order to study the response of the new sensitive material to $\mathrm{CO}_{2}$, some glass slides were coated by a casting method. After 60 minutes of gelation, the layers were ready for sensing applications. The coated films, which presented a faint yellow color, were very homogeneous, and were characterized by absorption spectroscopy in the visible range and also by refractometry

\subsection{Absorption measurements}

For characterization of the sensing layers, the setup showed in figure 1 was used. The previously coated samples were placed in a test chamber and illuminated by a Violet LED $(\lambda \mathrm{c}$ at $410 \mathrm{~nm})$ through a $4 \mathrm{~mm}$-diameter multimode fiber bundle. The transmitted signal was collected through identical fiber bundle and carried for detection. An Ocean Optics USB4000 spectrometer, connected to a PC, was used for the absorption measurements. Using this setup the sensing films were tested in the concentrations range from $0 \%$ to $100 \% \mathrm{CO}_{2}$. This was achieved by using a flow control system fed with $\mathrm{CO}_{2}$ and Nitrogen inlets. At this stage, measurements were made in saturated moisture environment, to avoid cross sensitivity with humidity level. The membrane thickness was approximately $9 \mu \mathrm{m}$. Figure 2 shows the LED transmitted spectrum after crossing the test camber with different $\mathrm{CO}_{2}$ concentrations, in the range $0 \%$ to $100 \%$.

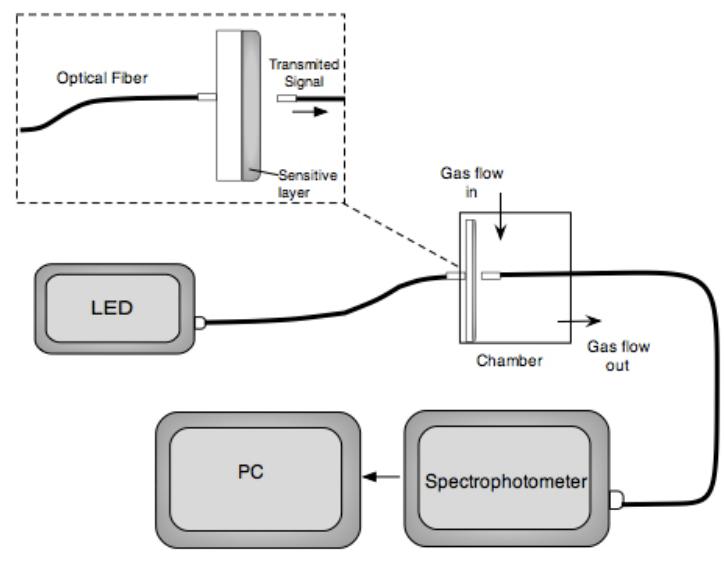

Figure 1. Setup for absorption measurements

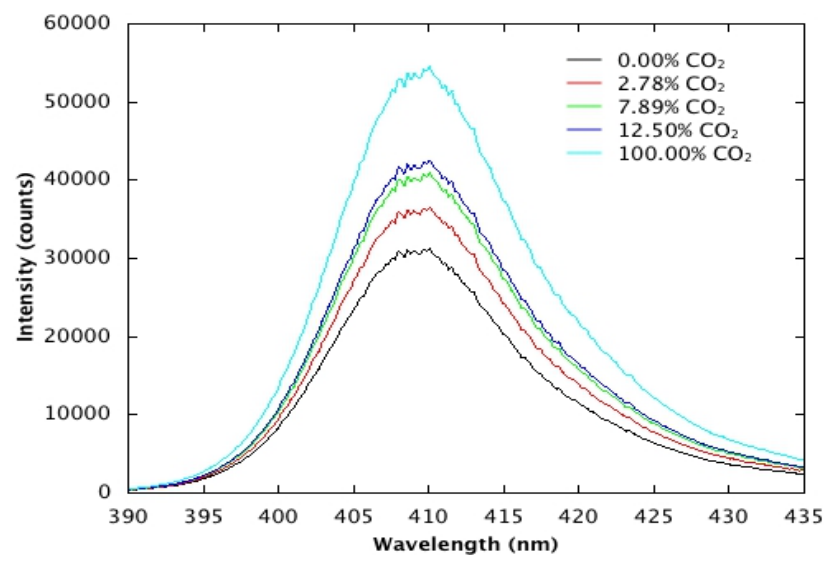

Figure 2. Transmitted spectrum for different $\mathrm{CO}_{2}$ levels

In figure 3 the response of the sensor to dynamic changes in $\mathrm{CO}_{2}$ concentration can be observed. From this data a calibration curve for the lower range of $\mathrm{CO}_{2}$ levels, $\sim 15 \%$, could be obtained that is showed in figure 4 . The results show a clear increase of transmittance with the increment of the $\mathrm{CO}_{2}$ concentration showing that indeed the presence of the gas in the membrane is causing changes in the electronic distribution of the sensitive membranes through protonation induced by the presence of carbonic acid. Observing the calibration curve in figure 4 it can be seen that the sensor is 
more sensitive in the lower concentration range and that a saturation effect occurs at higher concentrations. The transmittance variation for the range between $0 \%$ and $15.25 \%$ was approximately $40 \%$. In this range, the highest sensitivity was observed between $0 \%$ and $5.41 \%$, where a transmittance variation of $\sim 25 \%$ was noticeable. A full range response was also evaluated. From $0 \%$ to $100 \%$, the transmittance increased $\sim 70 \%$, confirming the saturation of the membrane. From the dynamic behaviour displayed in figure 3, a response time of $\sim 51 \mathrm{~s}$ could be estimated. Also from this data, a resolution of $\pm 0.15 \%$ was calculated considering a minimum detectable signal of two times the standard deviation. In the figure 3 is also noticeable the reversibility of the response from $15.25 \%$ to $0 \%$ and from $100 \%$ to $0 \%$. The repeatability of the response was also observed by performing the same experiment three times, the results obtained agreed within the measured standard deviation.

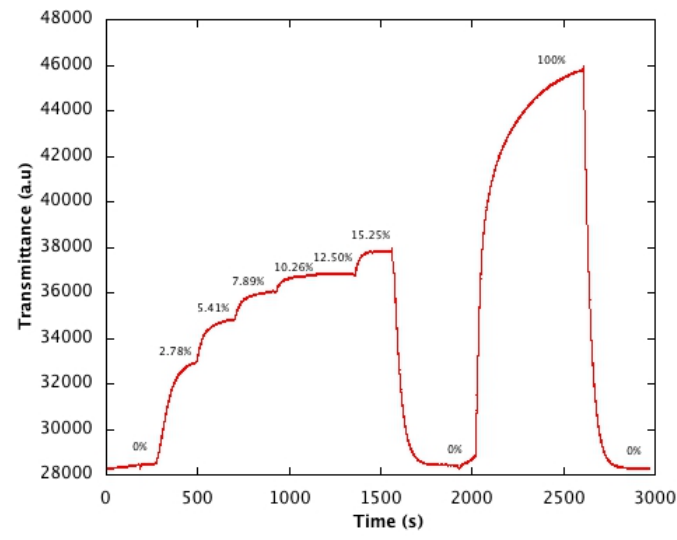

Figure 3. Sensor response to dynamic changes in $\mathrm{CO}_{2}$ concentration.

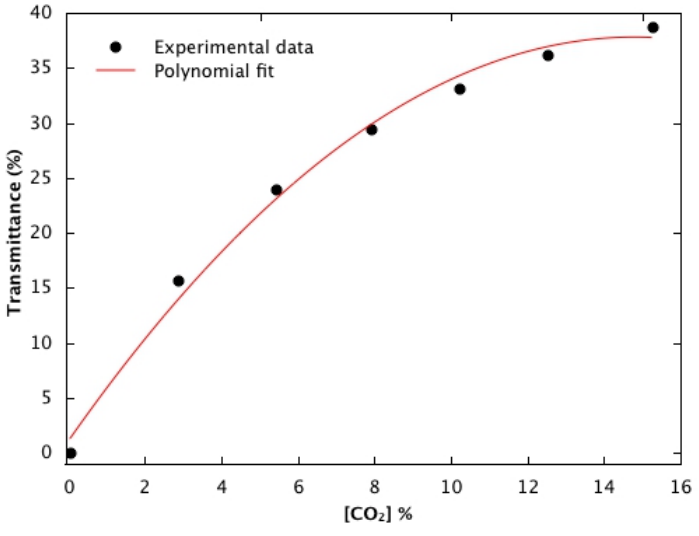

Figure 4. Calibration curve obtained for the $0 \%$ to $15 \% \mathrm{CO}_{2}$ concentration range.

\subsection{Refractometric measurements}

The refractometric changes in the sensitive layer where measured in the setup showed in figure 5. The coated glass substrates were placed in a test chamber and attached to the end face of an optical fiber inserted into a ceramic ferrule. To avoid undesirable reflections the interface between the fiber and the sample was filled with index matching oil. In this situation two low-finesse Fabry-Perot cavities were formed by the different material interfaces. The first cavity was produced by the reflections in the fiber/substrate and substrate/film interfaces. It had a thickness of approximately $1 \mathrm{~mm}$ and a refractive index of 1.489 at $1550 \mathrm{~nm}$. The second cavity was formed by the reflections in the substrate/film and film/external medium interfaces. For the refractometric measurements a layer with increased thickness was necessary, in other to allow the observation of at least a period of the interferometric pattern. In the present case, a layer with a thickness of $\sim 30 \mu \mathrm{m}$ was produced. This setup resulted in the observation of two-overlapped interferometric patterns at the fiber output (Figure 6). The larger substrate cavity resulted in a pattern with a very short wavelength modulation period $(0.80 \mathrm{~nm})$ while the thinner film cavity displayed a much larger periodic modulation $(\sim 30 \mathrm{~nm})$.

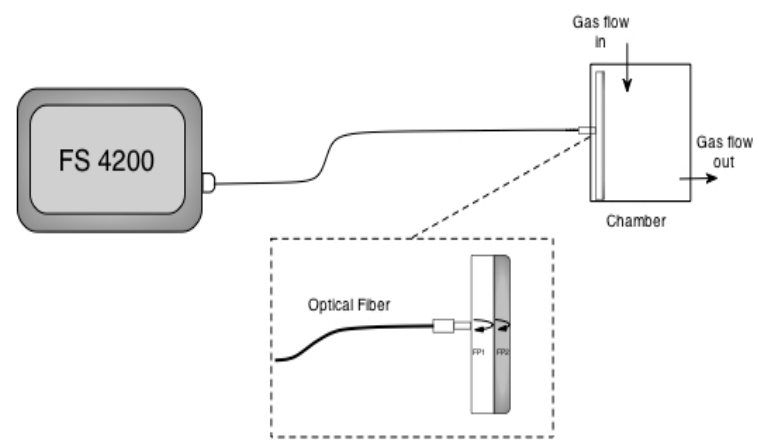

Figure 5. Interferometric setup for evaluating the sensing film refractive index taking advantage of the Fabry-Perot cavities formed by the substrate and the sensitive layer

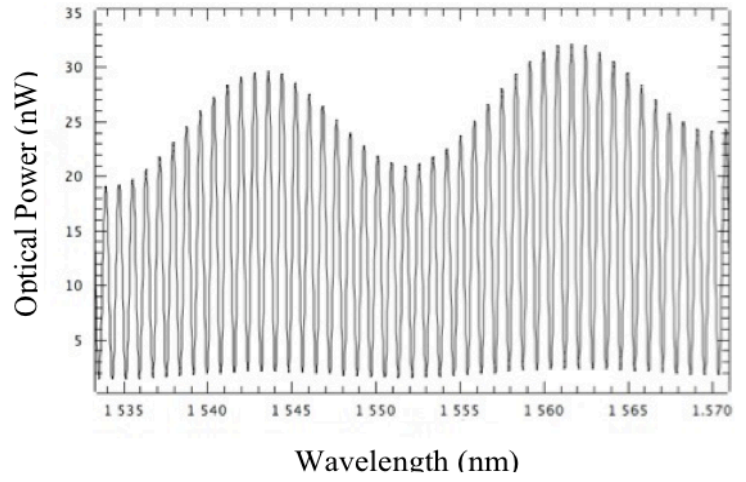

Figure 6. Typical interferometric output spectrum of the dual cavity setup 
The interferometric output was monitored using a scanning laser unit from Fibersensing (FS-4200, 1520-1590 nm, 1 pm max. resolution) with modified software. The software separates the signal of the sensing film (longer period) using an envelope detector. Because the membrane refractive index variations result in changes in the wavelength of this interferometric pattern, the software was designed to monitor the peaks and valleys related to the sensing signal. Figure 7 shows the behavior of the envelop channeled spectrum when the film was submitted to different $\mathrm{CO}_{2}$ concentrations. It is visible a wavelength shift of the interferometric pattern of about $35 \mathrm{~nm}$, which was observed when changing the $\mathrm{CO}_{2}$ concentration from $10 \%$ to $30 \%$. A small change is also noticeable between $0 \%$ to $10 \%$. The sensing film was tested in the concentration range from $0 \%$ to $90 \% \mathrm{CO}_{2}$. At this point, and to avoid any cross-sensitivity with humidity, both gases were thoroughly bubbled trough water to become saturated with humidity. The Figure 8 shows the calibration curve obtained for the $0-90 \%$ concentration range.

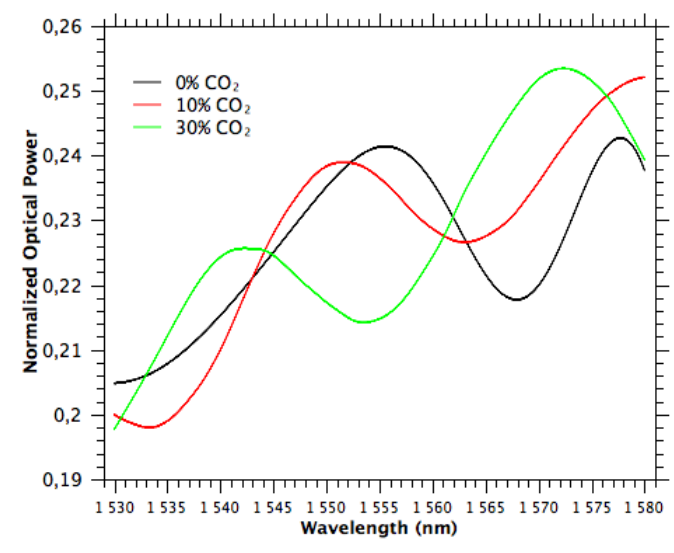

Figure 7. Interferometric spectra related to three different $\mathrm{CO}_{2}$ concentrations.

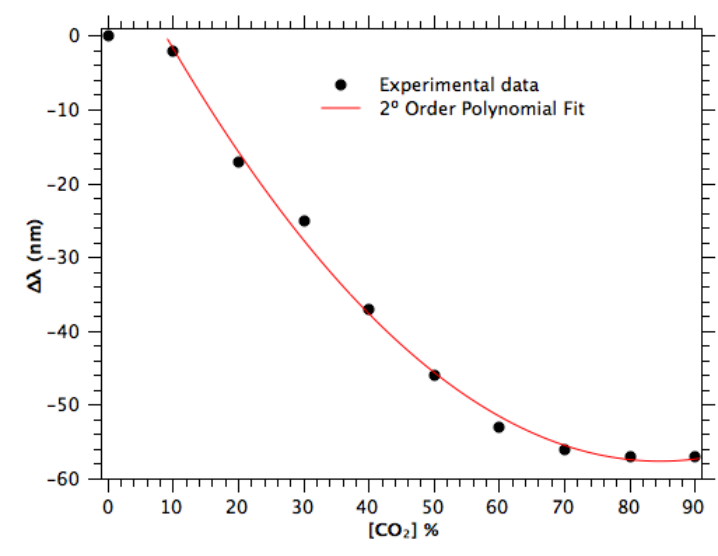

Figure 8. Response curve for a $\mathrm{CO}_{2}$ concentration range between $0-90 \%$

A nonlinear response was observed that could be fitted with a second order polynomial curve. A high sensitivity range could be established between $10-60 \%$, in which it is noticeable a wavelength shift of $51 \mathrm{~nm}$. Above $70 \%$ the membrane response becomes saturated. From this data, assuming that the membrane thickness remains unchanged and using a two wave interferometer approximation, it is possible to estimate roughly a refractive index variation of 0.045 RIU for the 0 $90 \% \mathrm{CO}_{2}$ range. Response time and reversibility were also studied. The best response time observed was approximately $3 \mathrm{~min}$. Also it was observed that refractive index change was not fully reversible, probably due to much larger recovery times. In this configuration, the response time and the stability of the response were not optimal due to the increased thickness of the layer $(\sim 30 \mathrm{um})$ that increased the diffusion time of the gas in the hydrogel matrix. At this stage further studies are underway, to understand the role of the immobilization matrix in the observed refractive index changes. Nevertheless, the results indicate the possibility of $\mathrm{CO} 2$ detection by refractometric measurements.

\section{CONCLUSIONS}

In this work, a polymer based sensitive layer for carbon dioxide measurement was presented. The sensing layer was based on the acid-base equilibrium of phenol and of its derivative p-nitro-phenol which, in the presence of $\mathrm{CO}_{2}$, are prone to protonation introducing absorption and refractive index changes. The new sensitive layers were casted on glass slides and characterized using a LED source $(\lambda c$ at $410 \mathrm{~nm})$ and an Ocean Optics USB4000 spectrometer. The layers were tested in the full concentrations range. The results show the increase of transmittance with the increment of the $\mathrm{CO}_{2}$ level. The membrane presents a non-linear response. The transmittance variation for the range between $0 \%$ and $15.25 \%$ was approximately $40 \%$. The dynamic behaviour was also analysed in this range. The determined response time was $51 \mathrm{~s}$ and a resolution of $0.15 \%$ was estimated. Some preliminary results indicate also that in the presence of the analyte the film refractive index is changed. The sensitive membranes were tested in an interferometric setup. $\mathrm{A} \mathrm{CO}_{2}$ dependent refractive index change of $\sim 0.045$ RIU was observed, in the $0 \%-90 \% \mathrm{CO}_{2}$ concentration range. These results show the viability of a carbon dioxide optical measurement using the phenol-based membrane with the application of colorimetric or refractometric based configurations. 


\section{ACKNOWLEDGEMENTS}

This work was supported by "Fundação para a Ciência e Tecnologia" (Project Aquamonitor. n. ${ }^{\circ}$ FCOMP-01-0124FEDER-013911 (Refa . FCT PTDC/AAC-AMB/112424/2009). Carlos Gouveia would like to acknowledge the financial support of FCT (SFRH/ BD/ 63758/2009).

\section{REFERENCES}

[1] R. Narayanaswamy and O.S. Wolfbeis, eds. Optical Sensors - Industrial Environmental and diagnostic Applications. Springer Series on Chemical Sensors and Biosensors, ed. O.S. Wolfbeis. 2004, Springer: Berlin.

[2] Rolfe, P., F. Scopesi, and G. Serra, Advances in fibre-optic sensing in medicine and biology. Measurement Science \& Technology, 2007. 18(6): p. 1683-1688.

[3] Wolfbeis, O.S. and B.M. Weidgans, Fiber optic chemical sensors and biosensors: A view back. Optical Chemical Sensors, 2006. 224: p. 17-44

[4] Baldini, F., et al., Continuous monitoring of gastric carbon dioxide with optical fibres. Sensors and Actuators BChemical, 2003. 90(1-3): p. 132-138.

[5] Baldini, F., et al., Carbon dioxide, oxygen and $\mathrm{pH}$ detection in animal adipose tissue by means of extracorporeal microdialysis. Optical Sensing Technology and Applications, 2007. 6585: p. U277-U283

[6] Mills, A., Optical Sensors for Carbon Dioxide and Their Applications. Sensors for Environment, Health and Security: Advanced Materials and Technologies, 2009: p. 347-370

[7] Wencel, D., et al., Ratiometric fluorescence-based dissolved carbon dioxide sensor for use in environmental monitoring applications. Analytical and Bioanalytical Chemistry, 2010. 398(5): p. 1899-1907.

[8] Pfeiffer, T.J., S.T. Summerfelt, and B.J. Watten, Comparative performance of CO2 measuring methods: Marine aquaculture recirculation system application. Aquacultural Engineering, 2011. 44(1): p. 1-9.

[9] Segawa, H., et al., Sensitivity of fiber-optic carbon dioxide sensors utilizing indicator dye. Sensors and Actuators B-Chemical, 2003. 94(3): p. 276-281.

[10] Amao, Y. and N. Nakamura, Optical CO2 sensor with the combination of colorimetric change of alphanaphtholphthalein and internal reference fluorescent porphyrin dye. Sensors and Actuators B-Chemical, 2004. 100(3): p. 347-351.

[11] Charpentier, F., et al., CO2 Detection Using Microstructured Chalcogenide Fibers. Sensor Letters, 2009. 7(5): p. 745-749.

[12] Orghici, R., et al., Fiber optic evanescent field sensor for detection of explosives and CO2 dissolved in water. Applied Physics B-Lasers and Optics, 2008. 90(2): p. 355-360. 